\title{
Expérimentalement religieux. Petite voie phénoménologique et pragmatique
}

Yves Meessen

\section{(2) OpenEdition}

1 Journals

Édition électronique

URL : https://journals.openedition.org/alter/2067

DOI : 10.4000/alter.2067

ISSN : 2558-7927

Éditeur :

Association ALTER, Archives Husserl (CNRS-UMR 8547)

\section{Édition imprimée}

Date de publication : 1 novembre 2020

Pagination : 227-243

ISBN : 978-2-9550449-6-4

ISSN : $1249-8947$

\section{Référence électronique}

Yves Meessen, «Expérimentalement religieux. Petite voie phénoménologique et pragmatique », Alter [En ligne], 28 | 2020, mis en ligne le 22 décembre 2020, consulté le 14 octobre 2021. URL : http:// journals.openedition.org/alter/2067 ; DOI : https://doi.org/10.4000/alter.2067

Ce document a été généré automatiquement le 14 octobre 2021

Revue Alter 


\title{
Expérimentalement religieux. Petite voie phénoménologique et pragmatique
}

\author{
Yves Meessen
}

Que chacun réalise pour soi ce dont il s'agit au contact d'une intuition vivante.

Ideen I, § 69, 223

Ce qui est visible n'a pas son origine dans ce qui apparaît au regard.

Hébreux 10,3

Comment parler de l'expérience religieuse sinon en s'exprimant «en première personne $»^{1}$ ? Cela ne veut pas dire que cette expérience soit purement et simplement subjective puisque la religion concerne la sphère sociale. Pourtant, emprunter la voie du sujet est la seule manière d'expérimenter la réalité à partir de l'intériorité. Je dis bien la réalité, la nôtre, celle de vous et moi, et non une autre réalité. Il en va de la découverte que nous sommes reliés les uns aux autres en deçà de l'écorce des choses telles qu'elles s'étalent devant nous. Le domaine religieux prend en effet ses racines dans la profondeur de la réalité. Il n'entre dans la catégorie des faits observables que par ses épiphénomènes : les pratiques, les rites et les espaces sacrés. Il est constatable socialement par le nombre d'adhérents qui se rassemblent ici plutôt que là. Mais ce type d'expertise manque ce qui caractérise fondamentalement l'expérience religieuse : une transformation du réel tel que nous le voyons, une métamorphose. Ce qui se transforme dans l'expérience religieuse, ce n'est pas seulement le sujet qui la vit, comme s'il se trouvait dans une sphère isolée du reste des hommes, mais le monde tout entier. Dans cette transformation, le rapport entre le sujet et le monde est médiatisé par la communauté religieuse à laquelle il appartient. Ceci me conduira à parler de Dieu à la troisième personne, en partant du rapport entre spiritualité et religion, pour finalement revenir à un «nous » qui se retrouve dans des récits partagés. Je vais donc parler de mon expérience religieuse en tant qu'elle me fait accéder à un dévoilement de la réalité, non pas comme quelque chose dont je serais le spectateur, mais comme un 
acteur engagé dans un processus de transformation. Voilà pourquoi mon approche couple deux méthodes : la phénoménologie et la pragmatique ${ }^{2}$.

2 Ce serait un malentendu de considérer l'épochè husserlienne comme une mise entre parenthèses du monde ordinaire. Est mise hors circuit l'identification de ce monde ordinaire à un monde objectif entièrement factuel. Dit autrement, le transcendantal ne quitte l'empirique que pour mieux en ouvrir les possibilités ${ }^{3}$. Ceci rejoint le courant pragmatique dans une de ses découvertes les plus fondamentales: la distinction entre le langage constatif et le langage performatif ${ }^{4}$. La réalité ordinaire y est abordée dans son devenir plus ou moins proche en tant qu'il propose ou exige une action à faire. Ainsi, que l'on soit en philosophie continentale ou anglo-saxone, il est possible de faire droit, non seulement à la choséité du réel, mais à son événementialité.

\section{Petite voie phénoménologique}

\section{Tâtonnement : découvrir l'abandon}

3 Pendant longtemps, j'étais convaincu que l'expérience religieuse nécessitait de rompre avec la vie ordinaire. Je croyais que je devais rentrer en moi-même en faisant taire tous mes sens pour attendre une révélation venue d'ailleurs. Cela ne suffisait pas de fermer les yeux. Il me fallait encore supprimer toutes les représentations et tous les bruits venant du monde sensible. Il me semblait suivre ainsi, de manière fidèle, les chemins pratiqués par les anciens. En effet, dans de nombreuses traditions religieuses, le renoncement au monde apparait comme la voie royale pour découvrir la vie intérieure.

Tant que tu as encore l'attention pour ce qui relève du corps, tant que ton intelligence tient compte des agréments extérieurs, tu n'as pas encore vu le lieu de la prière : tu es même loin de la voie bénie qui y conduit ${ }^{5}$.

4 Les ascètes du désert, tel qu'Évagre le Pontique (399 ap. J.-C.), n'envisagent pas de trouver Dieu ailleurs que dans la prière. Seule une purge de l'intelligence, non seulement de toute passion mais aussi de tout concept, rend apte à recevoir Dieu. La radicalité de cette méthode s'avère pleine d'embûches pour le méditant inexpérimenté. Ce n'est pas seulement par manque de radicalité, mais aussi par excès, qu'il peut manquer la cible. Ainsi en a-t-il été pour moi. Je me disais qu'il fallait arriver à l'extinction même de mon intentionnalité. Ne plus rien avoir sous les feux de mon intention, tel était mon objectif. Or, cet effort même était contreproductif. Vouloir ne plus vouloir, c'est encore vouloir quelque chose. Et, à la longue, cet exercice s'avère exténuant. C'est d'ailleurs là le salut de la méthode, mais je ne la savais pas. Après avoir pratiqué la prière silencieuse chaque matin durant une heure, et cela pendant des années, ma volonté s'affaiblit peu à peu jusqu'à devenir lasse. Cette lassitude, que je prenais pour une faiblesse, était pourtant ma force. Un beau jour - je ne sais plus quand -, mon vouloir a abdiqué. Il a dit: "Cette fois, c'en est trop. Je n'y arriverai pas. J'abandonne». À peine en étais-je arrivé là que, soudain, tout était différent. J'éprouvais expérimentalement que mon intelligence était prise en charge. Mon intention se portait çà et là, mais de manière détachée, c'est-à-dire sans être attachée à son objet. Sans que je ne sache comment, ma lassitude s'était changée en un «se laisser » (sich lassen). Je vivais le dessaisissement, la Gelassenheit, selon le beau mot de Maître Eckhart ${ }^{6}$. J'avais perdu le combat, mais il s'était changé en victoire. J'en étais tout heureux. Je bondissais de joie en jouissant de cette liberté intérieure. Dans ma 
naïveté, je pensais être arrivé. Mais combien je me trompais. Mon inexpérience me faisait prendre pour un état ce qui n'était autre qu'un acte. Or, qui dit acte, dit aussi passage de la possibilité à l'acte. J'allais découvrir que ce "laisser être", ou cette Gelassenheit, demandait une vigilance de tous les instants. J'avais seulement entrebâillé la porte d'une nouvelle modalité d'être-au-monde.

\section{2. Épreuve : recevoir le don}

Retomber dans l'effort du vouloir sans le vouloir. Vouloir ne plus vouloir mais ne pas savoir comment le vouloir passe au non-vouloir, voilà l'épreuve. Lorsque l'on devient attentif à cette vie de l'intentionnalité qui se situe en amont du domaine empirique, on commence à reconnaître les mouvements de la conscience. Je découvrais la différence entre mes productions internes et la perception de ce qui se donnait à moi. Pour le dire en termes kantiens, je discernais entre ma spontanéité et ma réceptivité. Il y a une manière de recevoir la « donation » qui lui permet d'apparaître en tant que telle. Cela suppose que je ne veuille pas aussitôt m'approprier ce qui se donne à moi pour le remplacer par le «concept » (Begriff vient de begreifen). Le concept s'occupe de ce qu'il peut circonscrire, ce qui est déjà figé dans l'espace et le temps. Jusqu'à un certain point, il ne laisse pas le temps fluer comme tel. Par le concept, je veux maîtriser ce qui me tombe sous la main. Dès lors, les mains occupées par ce que j'ai pris, je ne suis pas libre pour qu'une nouveauté puisse survenir. Bien sûr, l'entendement est nécessaire. Sans lui, il ne serait possible ni de se parler ni de produire les biens nécessaires à notre vie quotidienne. Encore faut-il le laisser être efficace dans son domaine propre. Dès qu'il s'agit d'inventer du neuf, le concept devient encombrant. En refluant de la choséité empirique, le factum, le déjà fait, vers sa possibilité d'être comme ceci ou comme cela, mon intelligence devient capable d'une donation nouvelle. Ou plutôt, elle laisse la donation s'actualiser comme telle. L'intelligence découvre qu'il est possible de s'insérer autrement dans ce qui arrive. De là, elle peut agir sur les événements.

6 L'exercice de l'attention se change peu à peu en vigilance ${ }^{7}$. De noétique, il devient éthique. Sur base de cette distinction entre prendre et recevoir, j'en viens à accueillir certaines intentionnalités et à en refuser d'autres. C'est peu dire qu'un tel discernement modifie considérablement la vie intérieure. En effet, ce que nous avons en vue se présente comme des possibilités à actualiser. Notre intention vers (regard) est aussi notre intention de (agir). Du coup, on peut en parler comme d'un exercice spirituel. En grec, la vigilance se nomme nepsis. Les pères neptiques sont les moines qui pratiquent la prière du cœur ${ }^{8}$. À force de pratique, celui qui est devenu vigilant (neptikos) en vient peu à peu à un état de paix intérieure, appelé hésychia. Le passage de la vigilance à la sérénité, de la nepsis à l'hésychia, est un saut qualitatif dans la vie intérieure. On quitte le registre de l'intelligence pour celui du cœur. Si je devais décrire ce qui change, je dirais que, dans le cœur, l'intelligence et la volonté ne font plus qu'un. En dehors, les deux facultés restent en lutte permanente. L'hésychia est un état fragile. Comme il dépend d'une habitude acquise, la moindre infraction à cette habitude l'expulse hors de la paix du cœur. Le neptikos l'apprend à ses dépens. L'habitus, ou l'hexis, produit en effet le sentiment du propriétaire, de celui qui possède son état par lui-même (habitus vient de habere, hexis de ekein). Cette présomption, ou orgueil, est l'origine de la chute. Mais, au moment où mon sentiment de paix se change en effroi, je ne comprends rien. Je suis désorienté, dans la déconvenue la plus totale. 


\section{Sauvetage : vivre le pardon}

7 Jusque-là, dans une certaine mesure, je pouvais encore concevoir la source de la donation comme un «fond sans fond » (Abgrund) impersonnel et neutre. Je serais ainsi un étant donné à lui-même, en même temps que retenu par ce fond, dans une " coappartenance » (Zusammengehörigkeit) ${ }^{9}$. Or, l'épreuve douloureuse de découvrir que l'avoir ne me maintient (main-tenir) pas connecté à ce fond me conduit plus profondément dans l'abîme. Je sombre, littéralement. Tout devient sombre. Comment retrouver le chemin de l'hésychia? Ce qui faisait mon assurance est aujourd'hui ma ruine. Que faire ? J'ai beau réfléchir. Cela ne me sert à rien. Je tente de reprendre les choses depuis le début. D'abord se détacher des passions et des concepts. C'est vite dit. Je suis tellement affecté par ce qui m'arrive que je ne fais plus qu'un avec cette passion qui me dévore. Si j'avais affaire à un problème objectif, je pourrais lui faire face. Mais, ici, ce qui fait problème n'est autre que l'ego lui-même, mon ego. Je suis auto-affecté par moi-même. Je pâtis mon propre état de propriétaire. Je veux l'impossible : n'être plus à moi, me démettre de moi, pour pouvoir vivre cette paix de la donation. Sich-haben, sichgeben : voilà l'antagonisme ${ }^{10}$. Inutile de tourner autour du pot, disons-le tout net : je fais l'expérience intime et indubitable qu'être moi n'est pas à moi. Ce n'est pas de la théorie. Je l'éprouve comme une évidence, en première personne, justement. Et j'en souffre. Cette souffrance me déchire de l'intérieur : le fond de moi est un autre. Je n'ose dire un autre ego. Je ne puis affirmer qu'il est propre ou qu'il est étranger. Il n'est ni l'un ni l'autre. Il serait dès lors erroné de le nommer alter ego. Je devrais plutôt dire que cet autre est la possibilité de moi-même. Sans lui, je ne suis pas. Une fois cela découvert, où en suis-je ?

Dans l'aporie. La voie est sans issue (a-poros). Je ne suis plus devant le gouffre, comme si je pouvais m'abstenir de sauter, mais déjà avalé par l'abîme. Parce que la possibilité même d'advenir ne m'appartient plus, que j'en suis dépouillé, tout avenir me semble compromis. Désespoir, tel est le sentiment de la situation ${ }^{11}$. Au creux de cette douleur, pourtant, une sorte de possibilité impossible me fait signe. Il me reste un très mince espoir, un fol espoir. M'accrocher à l'idée que cet autre, dont l'absente présence s'impose à moi, pourra m'aider. « C'est insensé, c'est de la folie », crie ma raison. « S'il n'y a plus que cette voie, il faut la tenter ", crie mon cœur. Se mettre à prier. Non pas entrer dans l'intériorité pour se mettre à marmonner des prières. Pas de la récitation, mais un cri d'appel. Lancer le véritable «au-secours, à l'aide» de quelqu'un qui se noie. Et là, expérimenter quelque chose jusque-là totalement inconnu. Découvrir que cet autre, que je n'osais nommer comme ceci ou comme cela, se soucie de moi. Il entend quand je l'appelle. «Je » rencontre un « Tu » et non plus seulement un «Cela » ${ }^{12}$. Ce « Je-Tu » ne se représente pas plus qu'il ne s'explique. Il est là. Je ne peux l'oublier. La rencontre est paradoxale. Effroi et joie s'entremêlent. Je me sens à la fois indigne et accueilli tel que je suis. Ma reconnaissance a deux facettes qui ne vont pas l'une sans l'autre: je me découvre gratuitement donné à moi-même et ingrat de vivre comme si ce n'était pas le cas. C'est dans une seule auto-affectation que j'éprouve le sentiment de commettre une injustice et que je me sens réconcilié par celui que j'offense ainsi. Tel est la force du pardon (per-donare). Du coup, comme Jacob combattant avec l'ange, ma défaite s'est muée en victoire ${ }^{13}$. J'ai découvert désormais qu'une Gelassenheit peut en cacher une autre. Il ne s'agit pas seulement de trouver la sérénité pour être en paix avec soi-même en renonçant au vouloir. Car, quoiqu'en dise Heidegger, il y va du rejet « de l'égoïsme coupable et l'abandon de la volonté propre à la volonté divine $»^{14}$. Il n'y a finalement de 
culpabilité que pour un «Je » qui reste seul avec lui-même, emmuré dans son moi. Ma culpabilité est ôtée car le « $\mathrm{Tu}$ » me pardonne en m'arrachant à ma volonté propre ${ }^{15}$. Tant que je me considère comme le possesseur de moi-même, mon intelligence et mon vouloir restent prisonniers de ma tyrannie. L'abdication de mon vouloir propre au vouloir divin est tout le contraire d'un esclavage. C'est ma libération, mon salut.

\section{Petite voie pragmatique}

\section{Deux foyers d'une ellipse}

9 Cet autre, dont j'ai expérimenté l'action en ma faveur, de manière intime, la religion l'appelle Dieu. Les hommes religieux lui bâtissent des temples et lui rendent honneur à travers des fêtes. Il est vrai que moi aussi, dès mon enfance, j'ai été initié à cette vie religieuse à travers le christianisme. Au départ, je suivais les us et coutumes de ma famille. Ensuite, lorsque je fus en capacité de choisir, je n'ai accepté de continuer ma pratique religieuse que parce qu'elle correspondait à mon désir de spiritualité. Pour désigner le rapport entre les deux, Jacques Derrida parle des "foyers d'une même ellipse ». S'opposant à Emile Benveniste qui réduit l'étymologie du terme religio à la seule acception de Cicéron (De la Nature des dieux, 2, 28): attention scrupuleuse ou relecture de sa vie (relegere) ${ }^{16}$, Derrida fait droit à l'autre source étymologique, celle de Lactance (Les Institutions divines, IV, 27, 3-16) : piété qui relie les hommes entre eux dans un même culte (religare) : "Quiconque ne reconnaîtrait ni la légitimité de ce double foyer ni la prévalence chrétienne qui s'est imposée à l'intérieur même de la dite latinité devrait refuser les prémisses même d'un tel débat $\aleph^{17}$.

Pour Jean Greisch également, la double étymologie fournit «la matrice» de toute réflexion sur la religion ${ }^{18}$. Ceci balise ma voie pragmatique. À savoir, l'expérience intime du divin, par relecture de ma vie et par recueillement, premier pôle de l'ellipse, peut difficilement se passer de mon rattachement à un peuple rassemblé pour le culte divin, second pôle de l'ellipse. Persévérer dans la religion chrétienne n'a pas été une option par défaut mais un véritable choix de ma part. J'ai découvert une profonde affinité entre ma propre démarche intérieure et la manière dont la Bible exprimait la révélation de Dieu. C'est cette affinité que je veux mettre maintenant en lumière. L'Ecriture m'apparait précisément sainte parce qu'elle est une voie de sainteté qui se propose à moi en même temps qu'à tous les hommes. Elle est le lieu scripturaire où des expériences subjectives trouvent leur place dans des événements qui concernent une vie sociale, à travers l'histoire d'un peuple, Israël. Or, ce qui est arrivé autrefois aux Hébreux peut être considéré selon deux regards tout différents. D'une part, je peux y reconnaître des faits historiques qui se sont effectivement passés et dont on peut constater la trace. D'autre part, je peux aussi y découvrir une expérience qui, bien qu'elle ait été vécue autrefois, est encore valable pour moi et pour nous aujourd'hui. Ces deux options ne s'annulent pas. Elles valent toutes deux dans leur domaine. Cependant, la première démarche tente de décaper le texte, en cherchant à mettre l'historique d'un côté et le fictif de l'autre, en privilégiant un langage constatif. En revanche, la seconde démarche s'accommode du texte tel qu'il est donné, dans son entremêlement historique et fictif, en privilégiant un langage performatif. Dans le premier cas, l'attitude critique est requise, d'où son nom: historico-critique. Dans le second cas, c'est l'attitude d'« auto-implication » (Self-involvement) qui prévaut ${ }^{19}$. 


\section{Performativité du texte et auto-implication}

11 La méthode historico-critique est bénéfique à la théologie au sens où elle la libère d'un regard objectiviste pour la placer devant sa tâche herméneutique: "retrouver l'expérience fondamentale d'un salut offert par Dieu en Jésus Christ $»^{20}$. Cela nécessite, continue Geffré, de "discerner les éléments fondamentaux de l'expérience chrétienne et de les dissocier des langages dans lesquels cette expérience s'est traduite ${ }^{21}$. Paradoxalement, pour ce faire, il ne propose pas de trier entre fait et fiction, mais, au contraire, de considérer leur entrelacs comme le lieu même d'une heuristique. Il promeut une nouvelle approche du texte en montrant que nous avons toujours déjà affaire à des «faits interprétés ». Seul le pli entre le factuel et le fictionnel permet la révélation d'une expérience dont la pertinence demeure actuelle. Autrement dit, l'écart provoqué par la fiction par rapport à l'historique est cela même qui permettra la réitération de l'expérience. Le langage métaphorique, parabolique, est capable de transfert. S'il veut préserver cette possibilité de transfert, le lecteur doit accepter de faire «comme si » les choses s'étaient passées telles qu'elles sont racontées, sans pourtant être dupe. C'est seulement par cette attitude non critique, explique Jean Ladrière, qu'il accèdera à la performativité du texte :

Lorsqu'un lecteur critique reprend un discours d'une force illocutionnaire F, il affecte ce discours d'une force illocutionnaire caractéristique de l'attitude critique. (C'est le cas d'un lecteur qui soumet le texte à une étude « scientifique ».) Par le fait même, il enlève à l'opérateur de force illocutionnaire du discours étudié son effectivité propre, se bornant à signaler les caractères (en indiquant par exemple qu'il s'agit d'un témoignage, ou d'une promesse, ou d'un conseil, etc.) Il ne s'agit pas à proprement parler d'une suppression mais d'une description désamorçante ${ }^{22}$.

Comme n'importe quel texte, les écrits bibliques sont les produits d'un geste illocutoire déterminé. En fonction du genre littéraire choisi, le texte fonde un " pacte de lecture " entre l'allocutaire et son destinataire ${ }^{23}$. Se tromper de genre revient à manquer ce pacte. Donc, à rendre la lecture inefficace, voire inutile. Tout texte biblique est performatif au sens où il fait ce qu'il dit. C'est d'ailleurs le sens du dabar: «Dieu dit et cela fut " (Genèse 1). La parole de Dieu ne dit rien sans en même temps faire quelque chose. Notons que, en finale de How to do Things with Words, Austin propose d'élargir la notion de performativité en distinguant le "performatif explicite", où la parole énoncée désigne simultanément l'acte ("la séance est ouverte»), à un sens du performatif où la parole énoncée incite à produire un acte qui sera suivi d'un effet (« veuillez fermer la porte»). La différence entre les deux s'exprime par l'emploi des termes «illocutoire » et " perlocutoire». Si l'on veut éviter la confusion, on peut parler de "performance» pour la performativité large ${ }^{24}$. Dans la Bible, les deux acceptions sont présentes, et cela, principalement, dans les textes narratifs. D'une part, le texte dit ce que Dieu fait au moment où il le dit, selon l'auto-implication des protagonistes du récit. D'autre part, le texte incite le lecteur à une auto-implication qui aura pour effet de découvrir que Dieu agit effectivement.

\section{Libération d'Égypte : paradigme de l'action de Dieu}

13 La libération d'Égypte est plus que la description de faits historiques. Elle est le véritable paradigme de l'action divine comme révélation: "Dieu nous a fait sortir d'Égypte à main forte et à bras étendu » (Deutéronome 26,8). Mais cela ne signifie pas que 
Dieu y soit présenté comme un deus ex machina. Ce sont bien des actions humaines qui ont eu lieu dans le cours même de la vie ordinaire: Moïse et Aaron interviennent auprès de Pharaon pour qu'il laisse s'en aller le peuple des hébreux. Bien sûr, avant cela, Moïse a fait l'expérience du Buisson ardent à l'Horeb (Exode 3-4). Mais, justement, le caractère propre de cette théophanie est d'avoir envoyé Moïse en mission afin que la force agissante de Dieu soit dévoilée à tous. L'histoire s'entremêle de fiction : les plaies d'Égypte sont là pour rehausser l'action divine par une sorte de vaste scénographie cosmologique. La création toute entière vient confirmer que la démarche de Moïse est approuvée et bénie par Dieu. Malheurs à ceux qui cherchent à la contrarier. Cette manifestation cosmique vire à l'apothéose lors du passage de la mer des roseaux. Les murailles d'eaux qui laissent passer le peuple à pied sec et se referment sur les chars égyptiens lancés à leur poursuite scellent la manifestation divine : Dieu a libéré son peuple à main forte. Tel est le message maintes fois répété au long de l'histoire d'Israël. L'écart entre fait et fiction s'est avéré efficace : Dieu agit et il le fait savoir. Il a agi et il agira encore. Or, finalement, ce sont les hagiographes (hagio = saint, graphô = écrire) qui ont raconté ce récit de cette façon. Ces hommes savaient pertinemment que s'ils s'étaient contentés de relater les faits sur un plan strictement historique, les lecteurs des années ultérieures n'auraient pu accéder à l'expérience vécue au moment de ces événements. Moïse est la figure emblématique d'un homme qui ne peut se satisfaire de l'esclavage d'Égypte. Il prend conscience qu'il peut faire quelque chose étant donné sa formation et sa proximité, quoique compromise, avec les hommes de pouvoir. Après avoir agi une première fois en faveur du peuple oppressé et avoir fui, sa conscience continue à le tarauder. Comme un buisson en flamme, il se laisse consumer de l'intérieur. Paradoxalement, il éprouve l'urgence d'agir car il ne peut fermer les yeux devant ce qu'il a vu : «J'ai vu la misère de mon peuple en Égypte et je l'ai entendu crier sous les coups des chefs de corvée » (Exode 3,7), et en même temps, il se sent trop faible pour accomplir ce qu'il sent devoir faire: «je n'ai jamais été doué pour la parole» (Exode 4,10$)^{25}$. Il ne va sortir de cette contradiction qui le consume qu'en se découvrant porté par une force bien plus grande que la sienne, celle de Dieu: "Je suis avec ta bouche » (Exode 4,12). Dans cet engagement, il sera secondé par son frère : « je sais qu'il a la parole facile » (Exode 4,14). Fort de cette solidarité, il osera affronter Pharaon. Cette audace est à elle seule un miracle au sens fort du terme (dunameis : geste de puissance). C'est un événement qui perturbe l'ordre établi. Mais cet ordre établi par un tyran est humain, trop humain. La nature elle-même se rebiffe. Quelque chose doit changer. La donation par l'intérieur, au creux de la conscience, provoque Moïse à l'action. La donation par l'extérieur, dans le delta du Nil, provoque des bouleversements. Une volonté de maîtrise excessive des canaux et des cultures a dévié ce qui devait se passer autrement. Dans les deux cas, par l'intérieur ou par l'extérieur, les choses se donnent telles qu'elles veulent être accomplies, à la fois dans leur poussée et leur finalité internes.

\section{Petite voie pragmatico-phénoménologique}

\section{Révélation comme mise en branle}

14 Les Écritures annoncent une révélation qui est en marche. Celui qui est à la source de la nature est aussi à la source des pensées et des actes de l'homme. Les deux convergent pour mener les temps vers leur accomplissement. Ce qui est arrivé aux protagonistes 
du récit, et qui a été mis en relief par la fiction, peut m'arriver à moi aussi. Aussi, lire " comme si » les choses étaient arrivées ainsi, selon une "seconde naïveté » dirait Ricoeur, m'a permis de découvrir l'expérience d'hommes qui ont éprouvé le dynamisme divin au creux de leur vie ordinaire. Les aspects extraordinaires du récit ne sont là que pour mieux mettre en évidence l'écart entre la possibilité de l'homme lorsqu'il constate ses moyens et l'effectivité de l'événement tel qu'il se produit. Du coup, le «comme si » fait passer du fictif à l'effectif. Il y a une performativité du récit historico-fictif. Mais pour qu'il y ait performance, la condition requise est le to perform.

Le propre d'une révélation, c'est précisément de ne pas offrir une sorte de vue achevée qu'il suffirait de recevoir dans une évidence simple, mais de donner seulement à celui qui la reçoit l'ébranlement nécessaire pour que, se mettant en marche, il puisse commencer à entendre ce qui est annoncé; c'est seulement dans la fidélité de la démarche que la révélation produit ses fruits ${ }^{26}$.

Si Moïse était resté à se consumer à petit feu, il n'aurait pas expérimenté la force de Dieu. La théophanie proprement dite n'aurait pas eu lieu. La théophanie ne se limite pas ponctuellement à l'épiphanie du buisson ardent mais à une longue séquence. C'est "l'arc-des-événements " ${ }^{27}$ englobant l'interpellation à l'Horeb, les péripéties de la libération, et le retour à l'Horeb. D'où, cette parole étrange que reçoit Moïse avant de se mettre en marche: «Je suis avec toi. Et tel est le signe que c'est moi qui t'ai envoyé : quand tu auras fait sortir d'Egypte mon peuple, vous rendrez un culte à Dieu sur cette montagne» (Exode 3,10). Le signe n'est pas donné d'avance. Seule sa donation est annoncée anticipativement. Lorsque le peuple sera arrivé à l'Horeb pour l'adorer, lui sera donné le signe que Dieu avait bien envoyé Moïse. Dieu ne se révèle pas dans une épiphanie, qui serait une présence objective, mais dans la dialectique de la promesse et de la mission (promissio-missio) ${ }^{28}$. C'est dire le caractère eschatologique de la révélation. Espérer pouvoir constater Dieu sur un mode spectaculaire est une illusion dont la Bible est la première à nous détromper. Dieu n'est pas une idole. Une fois que l'on a perçu cette modalité inhérente à la révélation de Dieu, il n'y a plus qu'à se mettre en branle.

\section{Les disciples d'Emmaüs : nos alter ego(s)}

16 Évidemment, c'est plus facile à dire qu'à faire. D'où vais-je recevoir la force de cette mise en marche ? La réponse est étonnante : du désir même qui m'habite de voir Dieu. Ce désir est un don. Il m'est donné en creux à la mesure de ma soif, de ma faim. Or, ce désir ne s'ouvrira au don qu'au travers d'une transformation décapante. Mais là aussi, le texte biblique me devance. Passons de l'un à l'autre Testament. Portons-nous d'un bond de la Pâque juive, commémorant la libération d'Égypte, à la Pâques chrétienne, fêtant la mort et la résurrection du Christ. Pessah, passage. Après avoir été un prédicateur et un thaumaturge errant avec quelques disciples à travers les territoires de Judée et de Palestine, Jésus a fini sa vie lamentablement. Livré par les chefs religieux au pouvoir romain, il a été cloué sur une croix. Deux disciples quittent Jérusalem, théâtre de cet événement morbide ( $\operatorname{uc} 24$ ). Désespérés, ils s'en retournent à leur village, appelé Emmaüs. Tout comme nombre de ceux qui suivaient Jésus, ils avaient mis leur espoir en lui pour qu'il les libère de l'occupant. Or, voilà que tout en marchant, un homme s'approche d'eux et se mêle à leur conversation. Cet étranger semble ignorer « ce qui s'est passé ces jours-ci » (Luc 24,18). En lui donnant leur version des événements, les disciples lui font également part de leur désespoir. Bien sûr, ils n'omettent pas le récit des femmes ayant vu des anges annonçant que Jésus est vivant, ainsi que des 
compagnons ayant trouvé le tombeau vide. Mais cela ne change rien à leur désarroi. Or, voici que, de manière inattendue, cet étranger leur remémore les Écritures : la Loi et les Prophètes. Annonçaient-elles autre chose que ce qui s'est passé ? «Ne fallait-il pas que le messie souffrît cela pour entrer dans sa gloire?» (Luc 24,26). Peu à peu, un autre regard possible sur les mêmes événements commence à poindre tandis que, au dehors, le soleil se couche à l'horizon. Il faut faire halte à l'auberge et se restaurer avant de se reposer afin de reprendre des forces pour le lendemain. Au moment du repas, l'étranger réitère le geste même de Jésus rompant le pain pour le leur donner. Juste à ce moment-là, coup de théâtre : tandis que leurs yeux s'ouvrent, le voyageur disparait à leur vue. Cette "apparition disparaissante", selon l'oxymore préféré de Vladimir Jankélévitch ${ }^{29}$, n'est pas qu'une théophanie parmi d'autres. Elle exprime la modalité même de la théophanie. C'est quand il n'y a rien à voir, quand le tombeau est vide, que les yeux voient enfin. Devant cette évidence fulgurante, les disciples peuvent courir raconter aux disciples restés à Jérusalem qu'ils ont vu le Ressuscité. La révélation est la relecture de l'événement de la mort à travers l'acte même du Christ. En se livrant d'avance à corps perdu, par le geste du pain rompu, Dieu se donne totalement et sans réserve. Par ce don lumineux, sans plus l'ombre de la moindre appropriation, la révélation opère un retournement phénoménologique magistral : elle fait reconnaître que le réel n'est pas dans l'écorce des corps, mais dans sa donation comme telle. Elle inverse tout. Ce qui apparait comme mort est en fait vivant, et ce qui apparait comme vivant est déjà mort. Voilà pourquoi, dans l'évangile de Jean, l'élévation de Jésus sur la croix est simultanément, dans le même acte, son élévation en gloire. La croix est la gloire car elle est la manifestation d'un don sans limite : l'amour.

Il y va dans ce récit d'une véritable performativité. Les disciples d'Emmaüs sont nos alter ego(s) car ils sont dans la même situation que nous. Jésus est mort. Jamais plus nous ne le verrons revivre avec son corps humain. Et pourtant, il nous reste la table de la parole et la table du pain. Ce sont les deux piliers des célébrations chrétiennes. Participer à la liturgie chrétienne consiste donc à redynamiser l'eschatologie. Autrement dit, le culte a une fonction performative. Il fait ce qu'il dit. Il rassemble des hommes et des femmes pour les incorporer à cette réalité en train de venir et dont la Parousie (Parousia $=$ présence) sera le don total.

\section{Apocalypse de la redondance}

Participer au culte n'est pas une finalité en soi. Ce n'est pas pour moi une convenance sociale. Ce n'est pas non plus une approbation sans réserve de l'institution ecclésiale. Comment le serait-ce puisque la Bible elle-même reste constamment méfiante par rapport à tout pouvoir religieux? Seul un retournement de la puissance en service est acceptable. Tout en n'étant pas dupe que l'on est encore loin du compte, je continue à participer au culte parce que c'est le meilleur moyen pour me constituer vigilant, neptikos, de plus en plus. Et cela n'a de sens, j'en suis expérimentalement convaincu, que si d'autres que moi le sont aussi. Car, l'eschatologie serait absurde dans la solitude. Dans la première partie de cette petite étude, j'ai mis en évidence la vie de l'ego à travers trois exercices : l'abandon, le don et le pardon. Il me reste ici à parler d'une quatrième modalité du don : «la redondance ». En français, le mot n'est pas très beau mais ce qu'il veut signifier, par contre, est absolument fondamental. Paradoxalement, 
vouloir saisir le don revient à le manquer. La parade à cet écueil consiste non à vouloir le don, mais à le recevoir en revivant l'acte donateur :

L'homme donc ne reçoit comme tel le don qu'en accueillant l'acte de donner, c'està-dire par répétition en donnant lui-même. Recevoir le don et le donner se confondent en une seule et même opération, la redondance $e^{30}$.

Pour que le don prenne corps en nous, il nous faut le rejouer pour «le performer à nouveau ». Non pas seulement dans le culte, mais dans la vie. En effet, le don du pain fait ce qu'il dit. À savoir, le quelque chose qu'il donne, c'est le corps donné, et donc, en fin de compte, c'est la donation elle-même. En recevant le corps du Christ, nous recevons en même temps d'être un seul corps. C'est le sens de la double épiclèse du canon eucharistique dans la tradition catholique. Il montre que la véritable performativité de la religion chrétienne consiste à faire advenir une réalité dans laquelle l'intersubjectivité dans le don est pleinement accomplie.

Pour y arriver, nul doute que les résistances ne manquent pas, à commencer par celle de mon petit ego. J'avoue, à la fin de cette confession de mon expérience, que je ne suis pas encore prêt à donner ma vie en pâture aux bêtes comme l'ont fait les martyrs. C'est même un euphémisme car je n'en ai pas le désir, tant cela me révulse et me fait horreur. Qui voudrait perdre son corps, et qui plus est, dans des circonstances affreuses? «En effet, nous qui sommes dans cette tente, notre corps, nous sommes accablés et nous gémissons, car nous ne voudrions pas nous dévêtir, mais revêtir un vêtement par-dessus l'autre, pour que notre être mortel soit absorbé par la vie » (2 Corinthiens 5,4). Pourtant, à relire les dernières pages de la Bible, il semble bien que le dévoilement (apo-calypse) ne se fasse pas sans un grand craquellement de l'écorce des choses telles que nous les voyons jusqu'à leur pulvérisation totale. Là encore, et bien plus que partout ailleurs, la fiction met en relief le grand combat qui a déjà commencé. Entre ceux qui veulent retenir le monde tel qu'il est dans son apparence, en se berçant de l'illusion que le réel est composé de substances individuelles solides, et ceux qui ont vu que toute réalité était fondée à même la donation, le combat est sans merci. Cependant, les armes ne sont pas les mêmes des deux côtés. À dire vrai, il n'y a d'armes que d'un seul côté, tant l'autre est désarmé. Comment le dire mieux qu'en faisant le récit d'un immense dragon voulant dévorer un petit enfant ${ }^{31}$ ? Et pourtant, le récit nous dit aussi, qu'après avoir répandu la terreur dans le monde pendant bien longtemps, le dragon sera vaincu. Il en est ainsi car l'appropriation n'est pas originaire, elle vient toujours après le don, comme la gangrène qui vient le ronger, sans toutefois pouvoir aboutir à ses fins: "Lorsqu'arrivera le renouvellement de toutes choses, la palingenèse » (palin : à nouveau, genesis : naissance, genèse, survenue), nous seront enfin dévoilées « des choses cachées depuis la fondation du monde » (Matthieu 19,28;13,35).

21 À qui trouverait que je suis indûment passé de la phénoménologie à la théologie, je répondrais sereinement qu'il n'est guère possible de parler de religion sans parler de Dieu (theo-logos). On m'objectera que tout dépend de la manière dont on en parle. En m'exprimant à la première personne, je ne pouvais faire autrement que m'impliquer dans l'évidence de ce que je vivais. Phénoménologiquement, dans l'intentionnalité, l'acte et le contenu ne font qu'un. Ce qui advient pourtant dans ma sphère propre, s'attestant lui-même en-deçà du langage, n'est pas transmissible à la sphère étrangère. Autrement dit, j'admets tout à fait que mes affirmations apothétiques restent hypothétiques pour mon lecteur ${ }^{32}$. Précisément, la rupture épistémologique entre une théologie philosophique et une théologie révélée ${ }^{33}$ ne se traduit pas par une distinction 
de corpus mais par une différence d'implication. C'est effectivement en elle, et non ailleurs, qu'a lieu la révélation. La distinction épistémologique n'est pas sémantique, comme on le pense habituellement, mais pragmatique. S'il n'en était pas ainsi, quiconque lirait un texte sacré, la Bible ou le Coran, recevrait aussitôt la révélation transmise par le texte. Le texte n'a donc pas de statut de révélation en lui-même mais pour le lecteur ou la communauté de lecteurs qui le reconnait tel. L'appartenance à telle ou telle religion s'actualise par cette reconnaissance commune. Le passage d'un statut à l'autre dépend donc de chacun, de l'auteur comme du lecteur. Elle est le fruit d'une décision personnelle. Voilà pourquoi, il me semble que, dans l'espace plurireligieux qui est le nôtre, nous avons tout intérêt à nous communiquer les uns aux autres ce qui, dans notre religion, nous fait vivre. Vouloir que cela reste tabou, selon une laïcité mal comprise, est la porte ouverte à tous les malentendus et à toutes les dérives. Notre tâche urgente est de désamorcer les graines de violence.

Vivre en religieux, en restant attentif aux deux foyers de l'ellipse, c'est pour moi être vigilant pour faire advenir une réalité qui ne demande qu'à éclore, à s'épanouir, comme les bourgeons des arbres avant l'été (Luc 21,30-31). La métaphore vaut en toutes saisons. Elle signifie que le « Royaume de Dieu » n'est pas un arrière-monde mais bien celui-ci même en tant qu'il n'est pas encore abouti. Il en va de ce royaume comme du levain enfoui dans quelques mesures de farine (Matthieu 13,33). Le monde est en train de lever, de se lever. Je désire participer à son aurore.

\section{NOTES}

1. N. Depraz, Comprendre la phénoménologie. Une pratique concrète, Paris, Armand Colin, 2006, p. 65 sq.

2. Je distingue méthodologiquement deux moments (I. Petite voie phénoménologique ; II. Petite voie pragmatique) pour mieux les unir dans un troisième moment (III. Petite voie pragmaticophénoménologique).

3. Voir C. Serban, Phénoménologie de la possibilité. Husserl et Heidegger, Paris, PUF, coll. « Epiméthée », 2016.

4. J. L. Austin, How to do Things with Words, Oxford, the Clarendon Press, 1962, trad. par G. Lane, Quand dire, c'est faire, Paris, Seuil, 1991.

5. Evagre le Pontique, Traité sur l'oraison, $\S 152$, dans Petite philocalie de la prière du cœur, traduite et présentée par J. Gouillard, Paris, Seuil, coll. « Points-Sagesse », 1979, p. 45.

6. M. Heidegger, Gelassenheit, Pfullingen, Günther Neske, 1957, dans Questions III, trad. par A. Préau, Paris, Gallimard, 1966, p. 159-182.

7. Voir N. Depraz, Attention et vigilance. A la croisée de la phénoménologie et des sciences cognitives, Paris, PUF, coll. « Epiméthée », 2014.

8. N. Depraz, "Pratiquer la réduction: la prière du cœur ", Laval théologique et philosophique, vol. 59, $\mathrm{n}^{\circ}$ 3, 2003, p. 503-519.

9. Voir par exemple : M. Heidegger, Identität und Differenz (1957), trad. A. Préau, «Identité et différence », Questions I, Paris, Gallimard, 1968, p. 262-270. 
10. K. Hemmerle, Thesen zu einer trinitarischen Ontologie, Fribourg-en-Brisgau, Johannes, $2^{e}$ éd., 1992, trad. par M. Dupuis, Thèses pour une ontologie trinitaire. Un manifeste, Paris, Ad Solem, 2014.

11. S. Kierkegaard, Traité du désespoir (1949), dans: Miettes philosophiques. Le concept d'angoisse. Traité du désespoir, trad. K. Ferlov et J.-J. Gateau, Paris, Gallimard, Tel, 1990, p. 337-496.

12. M. Buber, Ich und Du, 1923, trad. par G. Bianquis, Je et Tu, Paris, Aubier-Montaigne, 1938, rééd. 1992.

13. J.-L. Chrétien, «Comment lutter avec l'irrésistible », dans : Corps à corps. A l'écoute de l'œuvre d'art, Paris, Minuit, 1997, p. 11-24.

14. M. Heidegger, «Zur Erörterung der Gelassenheit » (1959), dans: Gelassenheit, Pfullingen, Günther Neske, repris dans : Aus der Erfahrung des Denkens (1910-1976), GA 13, 1983, trad. par A. Préau, Questions III, Paris, Gallimard, 1966, p. 183-225, ici, p. 187-188.

15. Il y a un malentendu très répandu sur l'articulation entre "volonté propre » et "volonté divine ». Ce qui est néfaste, ce n'est pas de vouloir librement soi-même en décidant ceci ou cela, comme si la "volonté divine " s'opposait à la nôtre sur le même plan. La volonté divine ne m'impose jamais ce que je dois faire. Parce qu'elle est transcendantale, la « volonté divine » est la condition de possibilité de la mienne. Du coup, plus je suis abandonné, plus je suis libre de vouloir vraiment moi-même. Cependant, tout en voulant ainsi, je reconnais que tout mon être, dans son acte libre, m'est donné par un autre.

16. E. Benveniste, Le Vocabulaire des institutions indo-européennes, t. 2, « Pouvoir, droit, religion », Paris, Les Éditions de minuit, 1969, p. 265 sq.

17. J. Derrida, Foi et savoir, Paris, Seuil, Points-Essais, 1996, rééd. 2001, p. 59.

18. J. Greisch, Le buisson ardent et les lumières de la raison, L'invention de la philosophie de la religion, t. I « Héritages et héritiers du XIX ${ }^{\mathrm{e}}$ siècle », Paris, Cerf, coll. « Théologie \& philosophie ", 2002, p. 15 sq.

19. Voir D. Evans, The Logic of Self-Involvement. A Philosophical Study of Everyday Language with Special Reference to the Christian Use of Language about God as Creator, (The Library of Philosophy and Theology), Londres, SCM Press, 1963; New York, Herder, 1969. Voir aussi, J. Ladrière, « Langage auto-implicatif, théologie et philosophie. À propos d'un ouvrage de M. D. Evans », Revue Philosophique de Louvain. Troisième série, Tome 65, n85, 1967, p. 115-122.

20. C. Geffré, "La théologie comme herméneutique" in Croire et interpréter. Le tournant herméneutique de la théologique, Paris, Cerf, 2001, p. 11-38, ici, p. 17.

21. Ibid.

22. J. Ladrière, L'articulation du sens, II. Les langages de la foi, Paris, Cerf, coll. "Cogitatio fidei ", 1984, p. 44.

23. J.-P. Sonnet, La parole consacrée. Théorie des actes de langage, linguistique de l'énonciation et parole de foi, Louvain-La-Neuve, Cabay, 1984, p. 74.

24. B. Cassin, «La performance avant le performatif ou la troisième dimension du langage » in B. Cassin et Carlos Lévy (dir.), Genèses de l'Acte de parole dans le monde grec, romain et médiéval, Turnhout, Brepols, 2011, p. 113-147.

25. Notons que la première proposition est attribuée à Dieu et la seconde à Moïse. Pourtant le «je» qui parle ne peut aussi être autre que Moïse dans les deux cas. Quel sens cela aurait-il si Moïse n'avait effectivement vu, auparavant, devant la souffrance de son peuple? Mais, le fait que l'hagiographe attribue cette vue à Dieu manifeste effectivement la transcendantalité de l'action divine par rapport à celle de l'homme.

26. J. Ladrière, L'articulation du sens, op. cit., p. 133.

27. P. Ricoeur, «Penser la création ", dans Penser la Bible (en collaboration avec A. Lacocque), Paris, Seuil, 1998, p. 56-104, ici, p. 70.

28. Voir J. Moltmann, Theologie der Hoffnung. Untersuchungen zur Begründung und zu den Konsequenzen einer christlichen Eschatologie, Munich, Kaiser Verlag, 1964 et J.-P. Thévenaz, Théologie 
de l'espérance. Essais sur les fondements et les conséquences d'une eschatologie chrétienne, Paris, CerfMarne, coll. « Cogitatio fidei », 1970, rééd. 1978.

29. V. Jankélévitch, Le Je-ne-sais-quoi et le Presque-rien, 2. La méconnaissance, Paris, Seuil, 1980.

30. J.-L. Marion, L'idole et la distance, Paris, Grasset, 1977, p. 213.

31. Voir Apocalypse 12,1-18.

32. Je fais ici référence à la distinction érigénienne entre ypothetica theologia et apothetica theologia reprise par Alain de Lille dans sa Summa quoniam homines I, éd. P. Glorieux, Archives d'histoire doctrinale et littéraire du Moyen Age, Volume 20, (1953), p. 121-122. Pour un développement de ce point, voir ma conférence de rentrée aux facultés catholiques de philosophie et de théologie de Lyon (Ucly), 16/09/2019: "Repenser la scientificité de la théologie : les promesses du langage », Theophilyon, Tome XXV, Vol. 1, 2020, p. 97-116.

33. Rappelons que l'articulation entre philosophie et théologie n'est pas à deux termes, mais à trois termes : philosophie, théologie philosophique et théologie révélée. 